\section{Zika Inquiries Made to the CDC-INFO System, December 2015-September 2017}

\author{
Tara Kirk Sell, Crystal Watson, Diane Meyer, \\ Michael R. Snyder, Sanjana J. Ravi, \\ Emma E. McGinty, Laura E. Pechta, Dale A. Rose, \\ Michelle N. Podgornik, Keri M. Lubell
}

\begin{abstract}
Author affiliations: Johns Hopkins Bloomberg School of Public Health, Baltimore, Maryland, USA (T.K. Sell, C. Watson, D. Meyer, M.R. Snyder, S.J. Ravi, E.E. McGinty); Centers for Disease Control and Prevention, Atlanta, Georgia, USA (L.E. Pechta, D.A. Rose, M.N. Podgornik, K.M. Lubell)
\end{abstract}

DOI: https://doi.org/10.3201/eid2605.181694

We examined Zika-related inquiries to CDC-INFO, the national contact center for the Centers for Disease Control and Prevention, to identify potential communication gaps. The most frequently asked questions related to travel or geographic location of Zika (42\% of all inquiries), information about laboratory testing (13\%), or acquiring a Zika test (11\%).

$\mathrm{A}^{\mathrm{n}}$ rapid increase in Zika virus disease transmission rates throughout Latin America in 2015, followed by transmission in some US states and outbreaks in several US territories, sparked widespread attention (1-3). We systematically examined Zika-related inquiries to the CDC-INFO system, the national contact center for the Centers for Disease Control and Prevention to determine public concerns and questions about Zika and potential communication gaps. We analyzed inquirer type, inquiry topic, and number of inquiries. In this article, "question" refers to the content of each call/email and "inquiry" to individual calls/emails, regardless of content. Inquiries may include $>1$ question.

The CDC-INFO Zika dataset contained 32,668 English-language inquiries (calls/emails) about Zika made from December 1, 2015 (when inquiries about Zika began to be tracked), through September 29, 2017 (when CDC's emergency activation for Zika response ended). We analyzed the number of inquiries over time using all database records and information on inquirers and topics using a 10\% simple random sample $(n=3,268)$. After an initial pilot process, 2 study authors coded notes made by operators for information on the types of inquirers and types of questions (4) (Appendix, https://wwwnc.cdc.gov/EID/ article/26/5/18-1694-App1.pdf).

We grouped inquirers into 3 different categories; however, most did not specify a category affiliation. The first category, the pregnancy group, made up $19 \%$ of all inquirers and was composed of pregnant women (10\%), women planning to become pregnant and their partners $(6 \%)$, and partners of pregnant women $(3 \%)$. The second category, clinicians, made up $14 \%$ of all inquirers. (CDC also initiated a separate hotline for clinicians during the Zika response; information from those calls was not included in this data analysis.) The third category, all other inquirers (67\%), included family members and parents $(8 \%)$; public health practitioners, students and educators, politicians and political staff, media, and salespeople (3\%); and inquirers who did not specify identity (56\%).

The most frequent questions (present in $42 \%$ of inquiries) were about travel or geographic location (geolocation) of Zika outbreaks (Table). Approximately $13 \%$ of all inquiries included questions seeking factual background information about laboratory testing, including how to obtain results, how to administer tests, how to interpret results, and criteria for testing. Questions about getting tested for Zika were present in $11 \%$ of inquiries. Questions related to transmission factors (e.g., incubation, persistence, immunity, semen, mosquitoes) were present in $9 \%$ of inquiries. Only $4 \%$ of inquiries included questions about health effects and related issues (e.g., potential harm to fetus, self, children) (Table).

Some types of questions were asked more frequently than others by certain groups. For example, the pregnancy group most frequently asked questions about travel or geolocation of the disease $(65 \%$ of all inquiries from this group), whereas clinicians most frequently sought information about tests (46\%) (Appendix Table 1).

Analysis of the number of inquiries over time showed 2 distinct peaks. The first occurred early in the response, with $\approx 4,000$ biweekly inquiries at the peak in late January/early February 2016, when news media coverage of the outbreak increased. The second occurred in late July/early August 2016, after local US transmission was confirmed, with nearly 2,000 biweekly inquiries (Appendix Figure 1). The number of inquiries by date for the 4 most frequently asked questions (about travel or geolocation of Zika, seeking information about tests, seeking to be tested for Zika, and transmission) generally reflected the same overall pattern as all inquiries. One exception was questions about travel/geolocation of the disease, which showed a third, smaller rise in volume in late 2016/ early 2017 (Appendix Figure 2). The frequency of 
Table. Percentage of inquiries with specific question topics to CDC-INFO, December 1, 2015-September 29, 2017*

\begin{tabular}{|c|c|}
\hline Question topic & $\begin{array}{l}\text { \% Inquiries with question topic, } \\
\qquad \mathrm{n}=3268\end{array}$ \\
\hline \multicolumn{2}{|l|}{ Information gathering } \\
\hline Transmission: includes persistence, presence in semen, mosquitoes, and immunity & 9 \\
\hline Incubation period & $<1$ \\
\hline Signs and symptoms & 2 \\
\hline Outbreak response processes & 3 \\
\hline Seeking information about diagnostic tests & 13 \\
\hline Seeking information about treatments, countermeasures, vaccines & 1 \\
\hline Clinician seeking clinical recommendation/assistance for a patient with Zika & 1 \\
\hline \multicolumn{2}{|l|}{ Information about risks } \\
\hline Health effects/issues: includes harm to self, fetus, pregnant woman, or child & 4 \\
\hline Health effects: specifically long-term reproductive effects & 4 \\
\hline Exposure: mosquito-related or sexual exposure & 2 \\
\hline Infection: asking if inquirer could have Zika & $\overline{1}$ \\
\hline Safety of protective actions: includes spraying or repellant & $<1$ \\
\hline \multicolumn{2}{|l|}{ Actions } \\
\hline \multicolumn{2}{|l|}{ Protective actions } \\
\hline What activities should be done to protect from getting Zika & 3 \\
\hline Waiting to get pregnant & 4 \\
\hline Safe sex practices & 1 \\
\hline Insect repellent/preventioning bug bites/mosquito control & 3 \\
\hline What action to take following possible exposure & 1 \\
\hline Acquiring a Zika test & 11 \\
\hline Travel and geolocation & 42 \\
\hline Actions for infected persons & $<1$ \\
\hline \multicolumn{2}{|l|}{ Other } \\
\hline Seeking access to materials/tools & 4 \\
\hline
\end{tabular}

questions about transmission, signs/symptoms, health effects, long term health effects, and insect management was significantly greater early in the outbreak, before local transmission was confirmed in the United States ( $p<0.05$ by $\chi^{2}$ test). Questions about waiting to get pregnant and geolocation were made significantly more often after local transmission occurred ( $\mathrm{p}<0.05$ by $\chi^{2}$ test).

Outreach to CDC-INFO might indicate that Zika messages reached intended target populations. Of those who volunteered demographic information, $44 \%$ were in the pregnancy group and so might have been made aware of heightened risks and sought more specific information about ways to reduce them. Results show that information needs were most intense at the time the threat emerged, although events during the outbreak, such as news of local transmission cases in the United States or new transmission routes, might also have increased public interest.

Inquiries made to CDC-INFO about Zika might represent potential information gaps from other sources. Inquirers most frequently asked about travel/geolocation of disease and testing, which was already included in CDC messaging and disseminated through various channels. Our findings could indicate that the information previously provided was perceived to be insufficient or difficult to locate or understand or that it was hard to keep up with changes in messaging during the response.

CDC-INFO records are a source of data that has been underanalyzed but that provides critical information about inquiries from the public about active efforts to obtain information from CDC. Our findings may help with future messaging efforts around infectious disease outbreaks by identifying topics of information that should be emphasized to improve public understanding.

\section{Acknowledgment}

The authors thank Rachel Ciccarone and Hoang-Kheim Ha for providing us the CDC-INFO Zika dataset and their technical assistance for this project.

This research was supported by CDC contract \#200-2016-92378

\section{About the Author}

Dr. Sell is a senior scholar at the Johns Hopkins Center for Health Security and an assistant professor in the Department of Environmental Health and Engineering at the Johns Hopkins Bloomberg School of Public Health. Her work focuses on improving public health policy and practice in order to reduce the health impacts of disasters and terrorism. 


\section{References}

1. Campos GS, Bandeira AC, Sardi SI. Zika virus outbreak, Bahia, Brazil. Emerg Infect Dis. 2015;21:1885-6. https://doi.org/10.3201/eid2110.150847

2. US Centers for Disease Control and Prevention. 2016 case counts in the US [cited 2018 Oct 26]. https:/ / www.cdc.gov/ zika/reporting/2016-case-counts.html

3. US Centers for Disease Control and Prevention. 2017 case counts in the US [cited 2018 Oct 26]. https:/ / www.cdc.gov/ zika/reporting/2017-case-counts.html

4. Slaughter L, Keselman A, Kushniruk A, Patel VL. A framework for capturing the interactions between laypersons' understanding of disease, information gathering behaviors, and actions taken during an epidemic. J Biomed Inform. 2005;38:298-313. https://doi.org/10.1016/j.jbi.2004.12.006

Address for correspondence: Tara Kirk Sell, Johns Hopkins Bloomberg School of Public Health, 621 East Pratt St, Ste 210, Baltimore, MD 21202, USA; email: tksell@jhu.edu

\section{Serologic Detection of Middle East Respiratory Syndrome Coronavirus Functional Antibodies}

\author{
Nisreen M.A. Okba, Ivy Widjaja, Wentao Li, \\ Corine H. GeurtsvanKessel, Elmoubasher A.B.A. \\ Farag, Mohammed Al-Hajri, Wan Beom Park, \\ Myoung-don Oh, Chantal B.E.M. Reusken, \\ Marion P.G. Koopmans, Berend-Jan Bosch, \\ Bart L. Haagmans
}

Author affiliations: Erasmus Medical Center, Rotterdam, the Netherlands (N.M.A. Okba, C.H. GeurtsvanKessel, C.B.E.M. Reusken, M.P.G. Koopmans, B.L. Haagmans); Utrecht University, Utrecht, the Netherlands (I. Widjaja, W. Li, B.-J. Bosch); Ministry of Public Health, Doha, Qatar (E.A.B.A. Farag, M. Al-Hajri); Seoul National University College of Medicine, Seoul, South Korea (W.B. Park, M.-d. Oh); Center for Infectious Disease Control, Bilthoven, the Netherlands (C.B.E.M. Reusken)

\section{DOI: https://doi.org/10.3201/eid2605.190921}

We developed and validated 2 species-independent protein-based assays to detect Middle East respiratory syndrome coronavirus functional antibodies that can block virus receptor-binding or sialic acid-attachment. Antibody levels measured in both assays correlated strongly with virus-neutralizing antibody titers, proving their use for serologic confirmatory diagnosis of Middle East respiratory syndrome.
The zoonotic introductions and ongoing outbreaks 1 of Middle East respiratory syndrome (MERS) coronavirus (MERS-CoV) pose a global threat $(1,2)$ necessitating continuous serosurveillance to monitor virus spread alongside the development of vaccine and antibodies as countermeasures. Both approaches require validated assays to evaluate specific antibody responses. Although MERS-CoV serologic assays have been developed (2-6), those detecting functional antibodies cannot be applied in all laboratories and can require Biosafety Level 3 (BSL-3) containment. Recombinant protein-based immunoassays are easier to operate and standardize and do not require BSL-3 containment. However, MERS-CoV protein-based assays developed thus far can only detect antibody binding and give no information on antibody functionality. The MERS$\mathrm{CoV}$ spike protein $\mathrm{N}$ terminal subunit (S1) contains 2 functional domains: the $\mathrm{N}$-terminal domain $\left(\mathrm{S} 1^{\mathrm{A}}\right)$, which binds sialic acid, the viral attachment factor; and the receptor-binding domain (RBD) $\left(\mathrm{S} 1^{\mathrm{B}}\right)$, which binds dipeptidyl peptidase 4 , the virus receptor $(7,8)$. Antibodies against those 2 domains can block MERS-CoV infection (9). Based on this fundamental knowledge, we developed 2 recombinant proteinbased functional assays.

First, we developed an S1-based competitive ELI$\mathrm{SA}$, a receptor-binding inhibition assay (RBI), to test for antibodies that block the interaction with dipeptidyl peptidase 4, the viral receptor (Appendix Figure 1, https://wwwnc.cdc.gov/EID/article/26/5/190921-App1.pdf). We validated the specificity of the assay for human diagnostics using serum samples from healthy blood donors, PCR-confirmed noncoronavirus-infected patients and non-MERS-CoVinfected patients (cohorts H1-H3) (Appendix Table $1)$. At a 1/20 dilution, none of the samples from nonMERS-CoV-infected humans showed a $\geq 50 \%$ reduction in signal $\left(\mathrm{RBI}_{50}\right)$ (Figure, panel $\mathrm{A}$ ), indicating a high specificity of the assay. MERS-CoV-specific RBI antibodies were detected in all the $90 \%$ plaque reduction neutralization assay $\left(\mathrm{PRNT}_{90}\right)$-positive serum samples of the PCR-confirmed MERS-CoV patients tested (Appendix Table 2, Figure 2). The percentage reduction in signal strongly correlated with neutralizing antibody titers (Figure, panel B). The $\mathrm{RBI}_{50}$ assay showed similar sensitivity to the PRNT ${ }_{90}$ assay.

Because the RBI assay is species-independent, we validated its ability to detect RBI antibodies in dromedaries. At a 1/20 dilution, none of the naive dromedary serum samples (10) reacted in the assay, whereas all samples from MERS-CoV-infected dromedaries (2) resulted in a $>90 \%$ reduction in signal (Appendix 